Journal of Clinical and Nursing Research

Research Article

\title{
Survey of the Sleep Quality of Medical Staff in a Grade A Tertiary Hospital during the Novel Coronavirus Pneumonia Outbreak
}

\author{
Qiugui Huang ${ }^{1 *}$, Jian $\mathrm{Yu}^{2^{*}}$, Linjun Liang ${ }^{3}$, Xiaofang Zhang ${ }^{4}$, Yuyao Liu ${ }^{5}$ \\ ${ }^{1,2}$ Isolation Ward, the First Affiliated Hospital of Jinan University, Guangzhou, China; \\ ${ }^{3}$ Precision Medical Diagnosis and Treatment Center, Zhuhai Hospital Affiliated to Jinan University, Guangzhou, \\ China; \\ ${ }^{4,5}$ Cancer Ward, the First Affiliated Hospital of Jinan University, Guangzhou, China
}

\begin{abstract}
This study aimed to investigate the sleep quality of medical staff in China who are combatting novel coronavirus pneumonia (COVID-19). To perform this, a survey of 127 medical staff from three separate wards (the general ward, isolation ward, and fever clinic) of a Grade A tertiary hospital in China was conducted. The survey questionnaire measured general characteristics and included the Pittsburgh Sleep Quality Index (PSQI). We consequently found that global PSQI scores differed significantly between the different wards $(\chi 2=44.561, \mathrm{P}<0.001)$. Whilst the medical staff in the general ward did not report any sleep disturbances, those in the isolation ward and the fever clinic both exhibited various degrees of sleep disturbance (global PSQI score $>7$ ). Medical staff in the isolation ward had the worst sleep quality (mean rank $=91.74)$, followed by those in the fever clinic (mean rank $=70.52$ ) and the general ward (mean rank $=39.10$ ), respectively. The primary sleep disturbances reported by the medical staff from the isolation ward included difficulty falling asleep ( 24 cases, $71.42 \%$ ), waking early or at night (28 cases, $80 \%$ ), and nightmares (18 cases, $51.42 \%$ ). In addition, $31.42 \%$ (11 cases) of the medical staff from the isolation ward had less than six hours of sleep a night, and $65.71 \%$ (23 cases) felt that their sleep quality was poor; meanwhile, for the staff from the fever clinic these ratios were $16.67 \%$ (seven cases) and $33.33 \%$ (14 cases), respectively. In contrast, among medical staff in the general ward, 98\% (49 cases) reported sleeping for more than six hours a night, and $96 \%$ felt that their sleep
\end{abstract}

quality was good. In conclusion, of the medical staff providing treatment for COVID-19, those in isolation wards and fever clinics are more likely to experience sleep disturbances.

Keywords: Novel coronavirus pneumonia, medical staff, sleep quality, Pittsburgh Sleep Quality Index.

Publication date: September, 2020

Publication online: 30 September, 2020

*Corresponding author: Jian Yu, yu38896@126.com

\section{Introduction}

The novel coronavirus pneumonia (COVID-19) outbreak, which commenced in China at the end of 2019 , is an ongoing situation affecting many countries worldwide. The primary source of infection is patients who are already infected with the novel coronavirus (SARS-CoV-2); further, asymptomatic patients may also contribute to infection ${ }^{[1]}$. The main route of transmission of the disease is through respiratory droplets and close contact; however, the disease can also transmit through aerosols when personnel are in relatively closed environments and are exposed to high-concentration aerosols for long periods ${ }^{[2]}$. In addition, SARS-CoV-2 can be extracted from feces and urine. In China, the COVID-19 outbreak has become the most serious public-health crisis since the establishment of the People's Republic of China and, to combat the epidemic, the government has adopted 
prevention and control measures designed for Class A infectious diseases ${ }^{[3]}$. Medical staff working in epidemic prevention and control and patient treatment experience high labor intensity, significant work stress, large infection risk, and heavy psychological burden ${ }^{[4]}$. These factors can increase the risk of developing sleep disturbances, which can directly lead to compromised immunity and, in turn, negatively affect prevention and treatment of SARS-CoV-2 infection ${ }^{[5]}$. This study investigated the sleep quality of hospital medical staff who are combatting the COVID-19 outbreak, seeking to identify influencing factors and associated characteristics in order to provide reference and base material that can contribute to the development of effective early interventions and necessary adjustments.

\section{Materials and Methods}

\subsection{Research subjects}

The ethics committee of our hospital approved the study. In order to carry out our research, we chose to conduct a questionnaire survey on the medical staff of a third-class and first-class hospital. Due to the outbreak in novel coronavirus, major hospitals are taking emergency prevention and control measures, so the scope of this investigation is a third-class and first-class hospital. To this end, a WeChat twodimensional code was generated using the mobile phone "Wen Juan Xing" software. This software is used by a large number of researchers in China. It is not only convenient to fill in and keep personal privacy, but also can save a lot of paper. The WeChat QR code was created during the outbreak in novel coronavirus to investigate the sleep quality of medical staff in a third-class A hospital. Therefore, this questionnaire is only used for clinical research and does not involve signatures. It was done voluntarily by medical staff. All questionnaires collected are confidential. We mainly investigated the classification of medical staff wards. The general ward group, isolation ward group and fever clinic group. The inclusion criteria are: (1) medical personnel working in hospitals are eligible to become medical practitioners or nurses; (2) Willing to agree to participate in the research. Exclusion criteria are: (1) suffering from heart, lung, liver or kidney diseases; (2) experience menstruation; And (3) suffering from depression, thyroid dysfunction, sleep apnea syndrome or sedation and hypnosis dependence.

\subsection{Research methods}

\subsubsection{General information questionnaire}

A general information questionnaire was created through referencing previous literature. This questionnaire contained items that focused on participants' gender, age, marital status, education, occupation, and the ward in which they worked.

\subsubsection{Pittsburgh Sleep Quality Index}

The Pittsburgh Sleep Quality Index (PSQI), designed by Buysse et $\mathrm{al}^{[6]}$, was used to assess the survey subjects' sleep quality. In 1995 , Liu et al ${ }^{[7]}$ tested the reliability and validity of this scale for Chinese samples, and found a Cronbach's $\alpha$ of 0.842 , indicating good reliability. The PSQI contains nine self-rated items (item five contains 10 subitems), and measures seven components: subjective sleep quality, sleep latency, sleep duration, habitual sleep efficiency, sleep disturbances, use of sleep medication, and daytime dysfunction. Items 1-4 ask respondents to enter values (such as the number of hours of sleep they get each night), while the remaining items are scored using a four-point scale (ranging from 0 to 3), with higher scores indicating greater symptom severity. The scores for each component are determined using the responses to the items, and are quantified into a range from 0 to 3. Scores of $\geq 2$ indicate poor or extremely poor quality or quantity of the component. The global PSQI score is then calculated as the sum of the scores for each component, giving a total score of 21 . The higher the global PSQI score, the worse the subject's sleep quality. A global PSQI score of $\leq 7$ suggests good sleep quality, and vice versa.

\subsection{Statistical analysis}

The statistical analysis of the data collected was performed using SPSS 23.0 software. Data were expressed as percentages or frequencies, and comparisons of these data were conducted using chisquare tests or Fisher's exact test. Alternatively, where necessary, the measurement data were expressed as means \pm standard deviations. Since they were not normally distributed and had inhomogeneous variances, the comparisons were performed via non-parametric tests. $\mathrm{P}<0.05$ was considered to indicate statistical significance.

\section{Results}

\subsection{General information}


A total of 127 medical staff participated in the survey, the effective recovery rate is $100 \%$, comprising 50 staff from the general ward, 42 from the fever clinic, and 35 from the isolation ward. The subjects consisted of 121 females and six males; of these, 110 were nurses and 17 were physicians; further, 39 were unmarried and 88 were married. There were no statistically significant differences between the three groups of medical staff regarding general information (gender, age, etc.; $\mathrm{P}>0.05$; Table 1).

\subsection{Individual Pittsburgh Sleep Quality Index scores for the medical staff in each ward}

There were statistically significant differences between the three groups for all PSQI components; that is, subjective sleep quality, sleep latency, sleep duration, habitual sleep efficiency, sleep disturbances, use of sleep medication, and daytime dysfunction $(\mathrm{P}<0.001)$. The primary sleep disturbances reported by the medical staff in the isolation ward included difficulty falling

Table 1. General information for the three groups of participants.

\begin{tabular}{|c|c|c|c|c|c|c|c|c|c|}
\hline & \multicolumn{2}{|c|}{ Gender } & \multicolumn{3}{|c|}{ Age (years) } & \multicolumn{2}{|c|}{ Marital Status } & \multicolumn{2}{|c|}{ Occupation } \\
\hline & Male & Female & $20-30$ & $30-40$ & Over 40 & Unmarried & Married & Physician & Nurse \\
\hline General Ward & 1 & 49 & 16 & 20 & 14 & 12 & 38 & 4 & 46 \\
\hline Isolation Ward & 1 & 34 & 15 & 13 & 7 & 16 & 19 & 4 & 31 \\
\hline Fever Clinic & 4 & 38 & 15 & 16 & 11 & 11 & 31 & 9 & 33 \\
\hline Total & 6 & 121 & 46 & 49 & 32 & 39 & 88 & 17 & 110 \\
\hline Chi-square Value & 2.711 & & 1.269 & & & 5.164 & & 3.467 & \\
\hline P Value & 0.241 & & 0.867 & & & 0.076 & & 0.172 & \\
\hline
\end{tabular}

\subsection{Sleep statuses of the medical staff in each ward}

Global PSQI scores differed significantly across the three groups of medical staff $(\chi 2=44.561, \mathrm{P}<0.001)$. Whilst the medical staff in the general ward did not report any sleep disturbances, both the isolation ward and the fever clinic groups exhibited various degrees of sleep disturbances (global PSQI score > 7). Medical staff in the isolation ward had the worst sleep quality (mean rank $=91.74)$, followed by those from the fever clinic (mean rank $=70.52$ ) and the general ward (mean rank $=39.10$; Table 2). asleep (24 cases, $71.42 \%)$, waking early or at night ( 28 cases, 80\%), and experiencing nightmares (18 cases, $51.42 \%$ ). In addition, $31.42 \%$ (11 cases) of the medical staff in the isolation ward reported having less than six hours of sleep a night, and $65.71 \%$ (23 cases) felt that their sleep quality was poor; meanwhile, in the fever clinic, these ratios were $16.67 \%$ (seven cases) and $33.33 \%$ (14 cases), respectively. In contrast, among medical staff in the general ward, 98\% (49 cases) reported sleeping for more than six hours a night, and $96 \%$ felt that their sleep quality was good (Table 2).

Table 2. Global and individual Pittsburgh Sleep Quality Index scores for the medical staff in each ward.

\begin{tabular}{lllllllll}
\hline Group & $\begin{array}{l}\text { Global PSQI } \\
\text { Score }\end{array}$ & $\begin{array}{l}\text { Subjective } \\
\text { Sleep Quality }\end{array}$ & Sleep Latency Sleep Duration $\begin{array}{l}\text { Habitual Sleep } \\
\text { Efficiency }\end{array}$ & $\begin{array}{l}\text { Sleep } \\
\text { Disturbances }\end{array}$ & $\begin{array}{l}\text { Use of Sleep } \\
\text { Medication }\end{array}$ & $\begin{array}{l}\text { Daytime } \\
\text { Dysfunction }\end{array}$ \\
\hline General Ward & $3.48 \pm 1.83$ & $0.78 \pm 0.51$ & $0.80 \pm 0.83$ & $0.26 \pm 0.49$ & $0.08 \pm 0.34$ & $0.94 \pm 0.31$ & $0.04 \pm 0.20$ & $0.66 \pm 0.72$ \\
Fever Clinic & $6.71 \pm 4.05$ & $1.36 \pm 0.76$ & $1.67 \pm 1.03$ & $0.64 \pm 0.76$ & $0.40 \pm 0.70$ & $1.24 \pm 0.58$ & $0.21 \pm 0.65$ & $1.33 \pm 0.98$ \\
Isolation Ward & $9.14 \pm 4.27$ & $1.83 \pm 0.86$ & $1.89 \pm 1.13$ & $1.06 \pm 0.84$ & $0.89 \pm 0.87$ & $1.31 \pm 0.53$ & $0.51 \pm 0.92$ & $1.69 \pm 0.99$ \\
Static Value & 44.56 & 35.89 & 14.99 & 23.06 & 31.25 & 15.98 & 9.46 & 24.02 \\
P Value & $<0.001$ & $<0.001$ & $<0.001$ & $<0.001$ & $<0.001$ & $<0.001$ & 0.009 & $<0.001$ \\
\hline
\end{tabular}




\section{Discussion}

In China, since January 23, 2020, the growth rate of newly diagnosed COVID-19 cases has continued to escalate, and the cumulative number of confirmed cases is rising daily ${ }^{[8]}$; this indicates that the COVID-19 outbreak is becoming increasingly serious in China, and especially in Hubei. To address this severe outbreak, provinces in China have activated first-level publichealth emergency responses ${ }^{[9]}$. Medical staff face a risk of infection when directly or indirectly contacting patients who may be infected with COVID-19. In addition, they experience heavy work stresses and psychological burdens when addressing unexpected emergencies and patient misunderstandings during treatment, which can lead to vicarious traumata, including sleep disturbances ${ }^{[10]}$.

The survey conducted in this study showed that sleep quality varies considerably across medical staff in isolation wards, fever clinics, and general wards, respectively. The medical staff in the isolation ward had the worst sleep quality, followed by those in the fever clinic. A possible reason for this is that medical staff in the isolation ward not only have a risk of infection, but also frequently work night shifts ${ }^{[11]}$, which can induce disruptions in circadian rhythms ${ }^{[12]}$. In this study, $31.42 \%$ (11 cases) of the medical staff in the isolation ward reported having less than six hours of sleep a night, and $65.71 \%$ (23 cases) felt that their sleep quality was poor; this group also reported sleep disturbances such as difficulty falling asleep, waking early or at night, and nightmares. Staff in the fever clinic showed the second-worst sleep status. In contrast, most medical staff in the general ward reported sleeping for more than six hours, and $96 \%$ felt that their sleep quality was good. Previous studies have suggested that sleep deprivation can affect various aspects of physical health, exerting an extensive influence on behavioral performance (e.g., advanced intelligence, learning and memory, emotions, and mental states), as well as physiological functions (e.g., cardiovascular and endocrine functions, the immune system, and energy metabolism $)^{[13]}$. Sleep deprivation can even cause irreversible damage to the body ${ }^{[14]}$.

By investigating the sleep quality of medical staff who are providing treatment during the COVID-19 outbreak, we found that medical staff in the isolation ward showed the worst sleep quality, followed by those in the fever clinic. The differences observed in our findings indicate that, to address these sleep- related issues, individualized improvement measures should be developed for each type of medical staff. I suggest that you can refer to the following methods. (1) Psychological interventions and counselling for medical staff who experience insomnia for more than two days. In addition, appropriate sedative-hypnotic drugs could be administered to such patients to reduce their sleep latency and increase their total sleep and habitual sleep durations, thereby promoting their physical health ${ }^{[15]}$. (2) Scheduling shifts to ensure staff can maintain healthy circadian rhythms. (3) Encouraging staff who have lost sleep the previous one or two nights as a result of their care commitments to compensate for this by either sleeping in the morning or sleeping early the next day. (4) Encouraging staff to try alternative approaches that can help them sleep. If a subject cannot fall asleep after 15 minutes, instead of lying awake in bed, he/she should do something that facilitates relaxation until he/ she feels sleepy. (5) Encouraging staff to release their emotions by communicating with family, friends, and colleagues.

As a result of the small number of medical staff from the isolation ward and fever clinic who agreed to participate in this research, there were strong biases in both the occupation and the gender data., there were strong biases in both the occupation and the gender data. To reduce the effects of these biases, the sample size could be enlarged, and a multivariate analysis could be performed.

\section{References}

[1] Wang D, Hu B, Hu C, et al. Clinical characteristics of 138hospitalizedpatientswith 2019 novelcoronavirus-in- fected pneumonia in Wuhan, Chinal [J]. JAMA, 2020. DOI: 10.1001/jama. 2020.1585. [Epub ahead of print].

[2] Zhu N, Zhang D, Wang W, et al. A novel coronavirus from patients with pneumonia in China, 2019 [J]. NEngl J Med, 2020,382(8):727-733.

[3] General Office of National Health Commission of the People's Republic of China, General Office of National Administration of Traditional Chinese Medicine. COVID-19 Diagnosis and Treatment Plans. 7th trial ed.; 2020.

[4] Xie Lu, Kang Hui, Xu Qing, et al. Sleep drives metabolite clearance from the adult brain[J]. Science, 2013, 342(6156): 373-377.

[5] Xiao H, Zhang Y, Kong D, Li S. The effects of social support on sleep quality of medical staff treating patients with Coronavirus Disease 2019 (COVID-19) in January and February 2020 in China. Med Sci Monit. 2020; 26: e923549. https://doi. org/10.12659/MSM.923921 [Epub ahead of print].

[6] Buysse DJ, Reynolds CF, Monk TH, Berman SR, Kupfer DJ. The Pittsburgh Sleep Quality Index: A new instrument for 
psychiatric practice and research. Psychiatry Res. 1989; 28(2): 193-213. https://doi.org/10.1016/0165-1781(89)90047-4

[7] Liu X, Tang M, Hu L, Wang A, Wu H, et al. Research on the reliability and validity of the Pittsburgh Sleep Quality Index. Chinese Journal of Psychiatry. 1996; 29(2): 103-7.

[8] China Center for Disease Control and Prevention Primary Response Situation Analysis and Risk Assessment Team for Pneumonia Infected in novel coronavirus. 2019 novel coronavirus Epidemic Progress and Risk Assessment[R/OL]. (2020-01-28) [2020-01-29] http://news medlive . cn/pul/info-progress/show165643 145. html.

[9] Wu J, Liu J, Zhao X, et al. Clinical characteristics of imported cases of COVID - 19 in Jiangsu Province: A multicenter descriptive studyl [J]. Clin Infect Dis,2020. DOI: 10. 1093/cid/ ciaa199. [Epub ahead ofprint].

[10] He Y, Wang Y, et al. Influences of COVID-19 on the sleep quality of frontline medical staff in Wuhan [J]. Medical Journal of Wuhan University, 2020. https://doi.org/10.14188/ j.1671-8852.2020.0159
[11] Wang J, Cheng YQ, Zhou Z, et al. Psychological status of Wuhan medical staff in fighting against COVID-19 [J]. Medical Journal of Wuhan University, 2020.DOI: 10. 14188/j. 1671 -8852. 2020. 0098. [Epubahead of print].

[12] Little A, Ethier C, Ayas N, et al. A patient survey of sleep quality in the Intensive Care Unit[J].Minerva Anestesiol, 2012,78(4):406-414.

[13] Grandner MA, Jackson NJ, Pak VM, Gehrman GR. Sleep disturbance is associated with cardiovascular and metabolic disorders. Sleep Res. 2012; 21(4): 427-33. https://doi.org/10.1111/ j.1365-2869.2011.00990.x

[14] Mullington JM, Haack M, Toth M, Serrador JM, Meier-Ewert HK. Cardiovascular, inflammatory, and metabolic consequences of sleep deprivation. Progr Cardiov Dis. 2009; 51(4): 294-302. https://doi.org/10.1016/j.pcad.2008.10.003

[15] Zheng X, He Y, Yin F, Liu H, Li Y, et al. Pharmacological interventions for the treatment of insomnia: Quantitative comparison of drug efficacy. Sleep Medicine. https://doi.org/10.1016/ j.sleep.2020.03.022 [In press]. 